\title{
The role of the hedgehog/patched signaling pathway in epithelial stem cell proliferation: from fly to human
}

\author{
PARISI Michael J, HAIFAN LIN ${ }^{1}$ \\ Department of Cell Biology, Box 3709, Duke University, \\ Medical Center Durham, N.C.27710,USA \\ e-mails:m.parisi@cellbio.duke.edu \\ h.lin@cellbio.duke.edu
}

\begin{abstract}
The hedgehog-patched (hh-ptc) intercellular signaling pathway has recently been shown to control the proliferation of epithelial stem cells in both Drosophila and vertebrates. Mutant and ectopic expression analyses in Drosophila suggest that the $\mathrm{HH}$ protein diffuses from the signaling cells to promote the proliferation of nearby ovarian somatic stem cells by antagonizing the suppression of its receptor PTC towards the CI transcription factor in the stem cells. Consequently, the transcription of CIdependent genes leads to stem cell proliferation. This regulatory pathway appears to function also in vertebrates, where defects in ptc cause basal cell carcinoma, tumors of epidermal stem cell origin. Basal cell carcinoma can also be induced by ectopic expression of Sonic hedgehog (shh) or Glil, the vertebrate homolog of ci. These studies suggest the conservation of the hh signaling pathway in controlling epithelial stem cell divisions among different organisms.
\end{abstract}

Key words: Stem cell, epithelia, hedgehog, patched, Drosophila, vertebrate.

\section{INTRODUCTION}

In the famous screen of Nüsslein-Volhard and Wieschaus for genes governing

1. Corresponding author. Phone: 919-684-3169; Fax: 919-684-5481 
hh/ptc in epithelial stem cell proliferation

Drosophila embryonic patterning, hedgehog (hh) and patched (ptc) were initially, shown to be involved in setting up the segmental boundary pattern of the fly embryo[1]. The $h h$ gene encodes a secreted ligand that can bind to its receptor complex composed of the transmembrane proteins on the surface of the target cells encoded by ptc and smoothened (smo) genes[2]. This binding releases the suppression of PTC towards a transcription factor encoded by cubitus interruptus (ci, a homolog of the mammalian Gli1 gene) through an intracellular signal transduction cascade, thus allowing several important genes including wingless ( $\mathrm{wg}$, a homolog of the mammalian Wnt genes), decapentaplegic (dpp, a member of the TGFb family), and ptc itself to be transcribed. The activation of these genes then specifies the fate of the target cells.

Studies in the past several years have revealed that the $h h / p t c$ signaling pathway is conserved in a number of postembryonic developmental processes in Drosophila and embryonic development in vertebrates. For example, during Drosophila metamorphosis, hh and ptc act to control cell fate decisions for patterning the central nervous system[3], legs, wings, (reviewed in[4, 5]) and eyes[6]. In vertebrates, the $\mathrm{hh} / \mathrm{ptc}$ pathway is involved in neurogenesis and limb pattering[7, 8]. More recently, the $\mathrm{hh} / \mathrm{ptc}$ pathway has also been shown to play a crucial role in regulating stem cell division and differentiation in adult Drosophila and mammalian epithelia[9-12], even though they appear to act on a different set of downstream genes. The hh/ptc signaling cascade in non-stem cells, especially with regard to components downstream of ptc inside the target cells has been reviewed in detail by Ruiz i Altaba[13]. This paper reviews the novel function of $h h / p t c$ in controlling stem cell divisions, with emphasis on the unique features of the $h h / p t c$ pathway in this process.

\section{hh/ptc pathway controls epithelial cell proliferation and differentia- tion in Drosophila oogenesis}

Drosophila oogenesis begins at the apical tip of the ovary in 15-17 functionally equivalent structures called the ovarioles (Fig 1a). Within each ovariole, oogenesis starts at the apical tip in a specialized structure called the germarium in which germline stem cells produce germline cysts. Each cyst then becomes enveloped by a layer of somatic epithelial cells called the follicle cells to form an egg chamber, which leaves the germarium and moves towards the oviduct as it develops further. To encapsulate new germline cysts, the somatic stem cells in the middle region of the germarium must undergo active divisions to generate a continuous supply of follicle cells[14] (Fig 1b). Recent work by Forbes et al. [9, 10] revealed that the division and differentiation of the somatic stem cells and their daughter cells are controlled by a group of non-mitotic somatic cells at the very tip of the germarium called the terminal filament cells through the $h h / p t c$ signaling pathway.

Forbes et al. $[9,10]$ first demonstrated a requirement for hh in regulating somatic cell proliferation and differentiation during oogenesis by showing that $h h$ is specif- 
ically expressed in the terminal filament cells. A hh enhancer trap line specifically stains the terminal filament; so did anti-HH antibodies. Functional evidence for $h h$ requirement comes from examining the effect of removing or ectopically expressing $h h$ in the germarium. When females homozygous for a temperature-sensitive hh mutation were raised at a permissive temperature to adulthood and then shifted to a restrictive temperature to remove $h$ activity in the ovary, this shift caused the severe underproliferation of follicle cells. In contrast, when hh was ectopically expressed as driven by a hsp70 promoter, this overexpression resulted in an increase in the number of follicle cells. These experiments were the first to establish that the $h$ signaling pathway plays a role in oogenesis and epithelial cell proliferation.

Fig 1.a. A schematic drawing of the Drosophila ovariole, which is composed of a string of egg chambers (EC) produced by the germarium (G).

b. A magnified view of the apical half of the germarium as boxed in panel a. hh is expressed in terminal filament cells (TF), while ptc is expressed in inner sheath cells (IC), somatic stem cells (SSC), and follicle cells (FC). GSC: germline stem cells; Cyst: germline cyst. The germline is shaded.

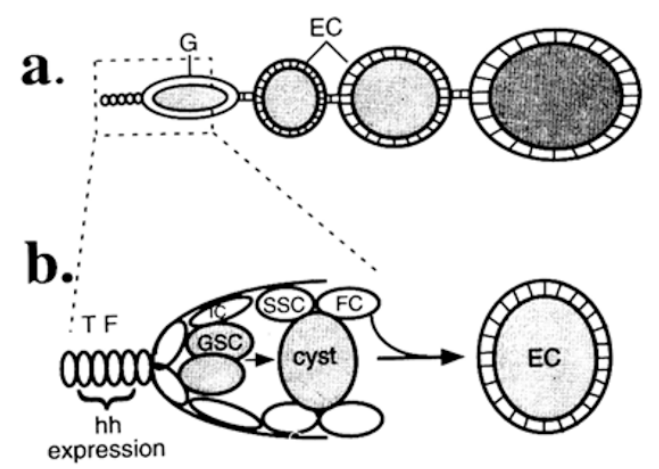

Given that the epithelium is a stem cell-derived tissue, hh may control the process by regulating stem cell division. This appears to be true since timed ectopic $h h$ expression experiments revealed that $h$ h acts on somatic stem cells and their immediate daughters[9].

Since $h h$ was known to interact with ptc in other Drosophila developmental processes, the function of ptc and other segment polarity genes in oogenesis were also tested[10]. Both the ptc-LacZ reporter and the anti-PTC antibody staining experiments showed that ptc indeed is expressed in a pattern complementary to the $h h$ expression pattern in the ovary. Loss of ptc function in clones of somatic stem cells and/or their daughter cells caused these cells to overproliferate, mimicking the effects of hh-overexpression. This suggests that ptc acts as a receptor molecule for the hh signal in controlling somatic stem cell division during oogenesis (Fig 2). Consistent with this, ectopic expression of ptc in the ovary showed no discernible effect on oogenesis, since the PTC protein is already present in the somatic stem cells and their immediate daughter cells without ectopic expression. These experiments provide substantial evidence that the functional relationship between $h h$ and $p t c$ is 
hh/ptc in epithelial stem cell proliferation

conserved in the epithelial stem cell system in the Drosophila ovary. These results also suggest that downstream targets of the ptc signal are insensitive to the concentration of PTC protein as long as it is above the threshold level, yet complete lack of PTC would fail to repress downstream target genes.

Fig 2. A simplified diagram of the $h h / p t c$ signaling pathway in regulating epithelial stem cell division in the Drosophila ovary. This pathway appears to function also in vertebrate epithelia.

Terminal Filament

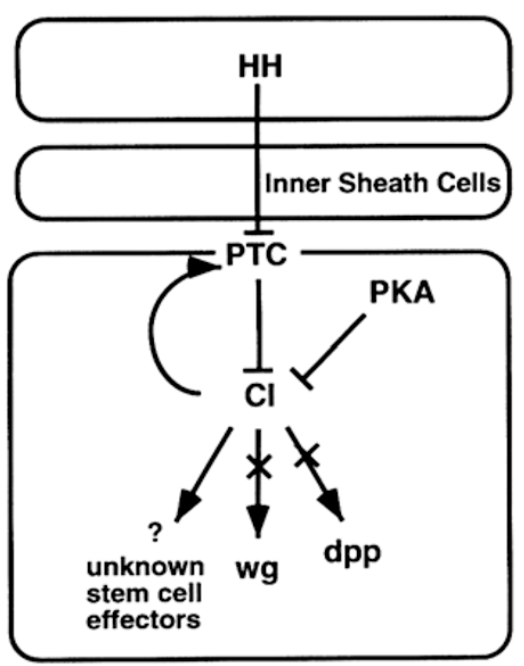

Somatic Stem Cells

Perhaps the most unexpected aspect of the $h \mathrm{~h} / \mathrm{ptc}$ pathway in the Drosophila epithelial stem cell system is the finding that $d p p$ and $w g$ are not the downstream effectors of the pathway as is the case in other developmental processes examined so far [10, Fig 2]. Although the ci expression suggests that it is under the control of the $h h / p t c$ pathway, its target genes no longer include $w g$ and $d p p$. It has been well documented in the Drosophila embryo and imaginal disc systems that wg and dpp are the effector genes of the hh/ptc signaling pathway, serving as the direct targets of the CI transcription factor. The finding that the epithelial stem cells do not require $w g$ and $d p p$ [10, H.L., unpublished results] suggests that there must be other effector genes more specific or relevant to the stem cell system. Thus, studies by Forbes et al. have shown not only the involvement of the hh/ptc pathway in controlling stem cell proliferation but also the divergence of downstream effector genes in this process. This role of the hh/ptc pathway in Drosophila epithelial stem cell division parallels its role in mammalian epithelial development as revealed by recent findings in vertebrate systems.

\section{$\mathrm{h} /$ patched as regulators of cell proliferation in mammalian epithelia}

The hh/ptc pathway has been characterized in vertebrate systems by isolating homologs, examining their expression profiles and manipulating their expression experimentally to examine the developmental consequences. For example, ectopic expression of Sonic hedgehog (Shh), a chick homolog of hh, caused mirror-like du- 
plications in limb structures that paralleled the mutant phenotype in Drosophila[8]. For another example, ptc in mice was shown to be expressed in tissues tightly linked to those expressing Shh[7]. Mice deficient in Shh had severe defects in embryonic development such as neurogenesis, axial determination, skeleton formation and limb development[15]. These results, together with the apparent similarity at the amino acid sequence level, suggest structural and functional conservation of the hh/ptc pathway in governing differentiation and patterning during mammalian embryonic development.

Recently, a growing body of evidence shows that the $S h h / p t c$ pathway also controls cell proliferation in vertebrate epithelia, a role similar to its function in controlling epithelial stem cell division during Drosophila oogenesis. Two recent reports[11, 12] have shown that the human homolog of ptc, $96 \%$ and $40 \%$ amino acid identity to the mouse and Drosophila Ptc proteins respectively, is mutated in individuals with an autosomal dominant disorder called Nevoid Basal Cell Carcinoma Syndrome (NBCCS). Consistent with the multiple developmental phenotypes of Drosophila mutants, defects in human ptc include a number of developmental anomalies which define its role in embryonic patterning. In addition, NBCCS individuals are prone to develop basal cell carcinomas (BCCs), as well as the induction of several other classes of tumors, suggesting ptc plays an important role in controlling cell proliferation in the affected tissues. The BCCs originate from pluripotent basal skin cells and tumors are associated with loss of heterozygosity at the human ptc locus. Since pluripotent basal skin cells by definition should be epidermal stem cells, the overproliferation of these cells in BCCs in ptc-deficient patients implies a role for ptc as a negative regulator of epidermal stem cell division as shown in Drosophila.

Further support for the $h h / p t c$ pathway in regulating basal cell division was provided by overexpressing Shh in transgenic mice bearing a Shh transgene driven by the keratin 14 promoter. This ectopic expression was sufficient to induce BCClike skin lesions in mice, thereby mimicking the loss of ptc function[16].

It is not surprising that several downstream targets of the $\mathrm{hh} / \mathrm{ptc}$ signaling pathway in Drosophila are themselves agents in tumorigenesis when mutated in vertebrates. Perhaps the most illustrative case is the role Gli1, a vertebrate homolog of ci, in incidences of various cancers including BCCs (reviewed in[13]). In all Drosophila developmental systems investigated, the transcription factor ci has been shown to be the downstream target of ptc signaling. In human, Glil was identified as a protooncogene causing incidences of glioblastomas (reviewed in[17]). Ectopic expression of Gli1 in frog embryos has recently been shown to cause the induction of BCCs, suggesting that Gli1 is potentially under the control of the $h h / p t c$ signal in regulating basal stem cell division. This hypothesis is consistent with the observation that in every case of human skin tumors with Shh expression, Gli1 is always expressed[18].

In addition to its role in BCCs, ptc has been shown to be mutated in 2 out of 14 sporadic medulloblastomas and in 2 out of 7 incidences of breast carcinomas ex- 
amined[19]. Also, activation of Wnt-2 and Wnt-3 has been correlated with tumor formation in the mouse mammary gland as shown by expression profiles and by mutant analysis[20]. Given that breast carcinomas and mammary gland tumors are of epithelial origin, it is likely that ptc and Wnt genes are involved in controlling epithelial stem cell divisions in this organ. Particularly, the involvement of Wnt-2 and Wnt-3 genes in this process suggests that other $w g$ homologs in Drosophila, instead of $w g$ itself, may be involved in regulating ovarian epithelial stem cell division. One future direction may be to examine which of these Wnt genes are actual targets of ptc regulation in Drosophila and mammals.

Although the use of Drosophila to dissect the molecular mechanisms of tumorigenesis is hindered by the short life cycle of the fly, screens for recessive mutations that cause overproliferation have been successfully conducted[21]. Genetic methods have been developed to screen specifically for tumor suppressors in the fly using Flp/FRT mediated genetic mosaics[22]. Given the known conservation between Drosophila and mammalian hh/ptc pathways as well as many other developmental pathways, the fly remains an effective model system for exploring the nature of stem cell division and overproliferation.

\section{ACKNOWLEGEMENTS}

M. P. is a recipient of a NIH postdoctoral fellowship (F32 HD08096-01A1). The writing of this review and the stem cell work in the Lin laboratory are supported by a NIH grant (R01HD33760), a David and Lucile Packard Fellowship, an ACS Junior Faculty Research Award and a March of Dimes Basil O'Connor Award to H.L.

\section{REFERENCES}

[1] Nusslein-Volhard C, Wieschaus E. Mutations affecting segment number and polarity in Drosophila. Nature 1980; 287:795-801.

[2] Alcedo J, Ayzenzon M, Von Ohlen T, Noll M, Hooper JE. The Drosophila smoothened gene encodes a seven-pass membrane protein, a putative receptor for the hedgehog signal. Cell 1996; 86:221-32.

[3] Bhat KM. The patched signaling pathway mediates repression of gooseberry allowing neuroblast specification by wingless during Drosophila neurogenesis. Development 1996; 122:2921-32.

[4] Perrimon N. Hedgehog and beyond. Cell 1995; 80:517-20.

[5] Ingham P. Signaling by hedgehog family proteins in Drosophila and vertebrate development. Current Opinions in Genetics Dev 1995; 5:528-34.

[6] Heberlein U, Moses K. Mechanisms of Drosophila retinal morphogenesis: the virtues of being progressive. Cell 1995; 81:987-90.

[7] Goodrich LV, Johnson RL, Milenkovic L, McMahon JA, Scott MP. Conservation of the hedgehog/patched signaling pathway from flies to mice: induction of a mouse patched gene by Hedgehog. Genes Dev 1996; 10:301-12.

[8] Riddle RD, Johnson RL, Laufer E, Tabin C. Sonic hedgehog mediates the polarizing activity of the limb. Cell 1993; 75:1401-16.

[9] Forbes AJ, Lin H, Ingham PW, Spradling AC. hedgehog is required for the proliferation and specification of ovarian somatic cells prior to egg chamber formation in Drosophila. Development 


\section{PARISI Michael Jand Haifan LIN}

1996; 122:1125-35.

[10] Forbes AJ, Spradling AC, Ingham PW, Lin H. The role of segment polarity genes during early oogenesis in Drosophila. Development 1996; 122:3283-94.

[11] Johnson RL, Rothman AL, Xie J, et al. Human homolog of patched, a candidate gene for the basal cell nevus syndrome. Science 1996; 272:1668-71.

[12] Hahn H, Wicking C, Zaphiropoulous PG, et al. Mutations of the human homolog of Drosophila patched in the nevoid basal cell carcinoma syndrome. Cell 1996; 85:841-51.

[13] Ruiz i Altaba A. Catching a Gli-mpse of Hedgehog. Cell 1997; 90:193-6.

[14] Margolis J, Spradling A. Identification and behavior of epithelial stem cells in the Drosophila ovary. Development 1995; 121:3797-807.

[15] Chiang C, Litingtung Y, Lee E, et al. Cyclopia and defective axial patterning in mice lacking Sonic hedgehog gene function. Nature 1996; 383:407-13.

[16] Oro AE, Higgins KM, Hu Z, Bonifas JM, Epstein E Jr., Scott MP. Basal cell carcinomas in mice overexpressing Sonic hedgehog. Science1997; 276:817-21.

[17] Collins VP. Gene amplification in human gliomas. Glia 1995; 15:289-96.

[18] Dahmane N, Lee J, Robins P, Heller P, Ruizi Altaba A. Activation of the transcription factor Gli1 and the Sonic hedgehog signalling pathway in skin tumours. Nature1997; 389:876-81.

[19] Xie J, Johnson RL, Zhang X, et al. Mutations of the PATCHED gene in several types of sporadic extracutaneous tumors. Cancer Res 1997; 57:2369-72.

[20] Nusse R, Varmus HE. Wnt genes. Cell 1992; 69:1073-87.

[21] Gateff E, Mechler BM. Tumor-suppressor genes of Drosophila melanogaster. Crit Rev Oncog $1989 ; \mathbf{1}: 221-45$.

[22] Xu T, Wang W, Zhang S, Stewart RA, Yu W. Identifying tumor suppressors in genetic mosaics: the Drosophila lats gene encodes a putative protein kinase. Development 1995; 121:1053-63. 